\title{
Influencia materna en el crecimiento predestete y características de la canal de corderos de pelo
}

\author{
Maternal influence on preweaning growth and carcass characteristics of hair lambs \\ Irma del Carmen García-Osorio ${ }^{1}$, Jorge Oliva-Hernández ${ }^{2 *}$, Mario Manuel Osorio-Arce $^{1}$, Glafiro \\ Torres-Hernández ${ }^{3}$, José Alfonso Hinojosa-Cuéllar ${ }^{4}$, Roberto González-Garduño ${ }^{5}$ \\ ${ }^{1}$ Colegio de Postgraduados, Campus Tabasco, Cárdenas, Tabasco, México \\ ${ }^{2}$ Campo Experimental Huimanguillo, Instituto Nacional de Investigaciones Forestales, Agrícolas y Pecuarias, Huimanguillo, \\ Tabasco, México \\ ${ }^{3}$ Colegio de Postgraduados, Campus Montecillo, Montecillo, Estado de México, México \\ ${ }^{4}$ Universidad Popular de la Chontalpa, Cárdenas, Tabasco, México \\ ${ }^{5}$ Unidad Regional Universitaria Sursureste, Universidad Autónoma de Chapingo, Teapa, Tabasco, México \\ *Autor de correspondencia: olivajh20@yahoo.com.mx
}

Artículo científico recibido: 01 de junio de 2015, aceptado: 23 de febrero de 2016

RESUMEN. El objetivo del presente estudio fue evaluar la influencia del tipo de nacimiento (TN) de los corderos y el número de parto (NP) de las ovejas sobre el crecimiento predestete, rendimiento de la canal (RC), composición corporal de los corderos y el comportamiento productivo de las ovejas. Se estudiaron 25 ovejas Blackbelly x Pelibuey y sus corderos, bajo un diseño factorial, los factores fueron TN (sencillo y múltiple) y NP (primípara y multípara). Los corderos se sacrificaron a los $56 \mathrm{~d}$ de edad. Las variables evaluadas fueron: peso vivo al nacimiento (PN) y al destete (PD), ganancia diaria de peso (GDP), peso de la canal y RC, peso y rendimiento de los cortes primarios. En las ovejas, se evaluó el peso al parto y al finalizar la lactancia, cambio de peso (CP), peso de la camada al nacimiento (PCN) y al destete (PCD). El TN afectó $(p<0.05)$ la GDP, PD, peso y RC de los corderos. En las ovejas, el tipo de parto (TP) y NP influyeron $(p<0.05)$ sobre el CP. El TP afectó $(p<0.05)$ el PCN y PCD. El TN afectó el crecimiento predestete, RC y composición corporal de los corderos, siendo los de nacimiento sencillo los que tuvieron mayor GDP, peso y RC. Mientras que las ovejas multíparas y ovejas con parto múltiple experimentaron el mayor CP negativo. En tanto que las ovejas con parto múltiple mostraron la mayor eficiencia productiva.

Palabras clave: Crecimiento, cordero, carne, lactancia, trópico húmedo

ABSTRACT. The aim of this study was to evaluate the influence of type of birth (TB) of lambs and number of parity (NP) of ewes on preweaning growth, carcass yield (CY), body composition of lambs and the productive performance of the ewes. Twenty five Blackbelly $\times$ Pelibuey ewes and their lambs were studied, in a factorial design; the factors were TB (single and multiple) and NP (primiparous and multiparous). The lambs were slaughtered at $56 \mathrm{~d}$ of age. The variables evaluated were: body weight at birth (BWB) and weaning (BWW), average daily wight gain (ADG), carcass weight and $C Y$ and weight and yield of the primal cuts. In ewes, body weight at lambing and at the end of lactation, change of body weight (CHBW), litter weight at birth (LWB) and at weaning (LWW) were evaluated. TB affected $(\mathrm{p}<0.05)$ ADG, BWW, carcass weight and $\mathrm{CY}$ of the lambs. In ewes, type of lambing (TL) and NP influenced $(p<0.05)$ CHBW. TL affected $(p<0.05)$ LWB and LWW. TB affected the pre-weaning growth, CY and body composition of the lambs, with the single lambs presenting higher ADG, carcass weight and $C Y$, while multiparous ewes and ewes with multiple lambing had the largest negative CHBW. However, ewes with multiple lambing had greater productive performance.

Key words: Growth, lamb, meat, lactation, humid tropic 


\section{INTRODUCCIÓN}

En los rumiantes, el crecimiento prenatal es influenciado por diversos factores, destacando el nivel de nutrición materna y la capacidad funcional de la placenta. Mientras que el crecimiento postnatal puede ser limitado por la nutrición materna y por factores ambientales (Bell et al. 1987). En el caso particular de los corderos, se sabe que el crecimiento predestete se afecta por la raza, tipo de nacimiento, sexo, edad al destete y el número de parto de la oveja (Hinojosa-Cuéllar et al. 2012). En los estudios en donde se evalúa la influencia del tipo de nacimiento del cordero y el tipo de parto de la oveja, sobre el crecimiento predestete de los corderos, solo se consideran los efectos principales, sin considerar la influencia de la interacción entre ambos factores (Ríos-Utrera et al. 2014).

Se han realizado pocos estudios en ovejas de pelo de clima cálido, en los que se evalúen los cambios de peso de las ovejas y sus camadas como indicadores de la productividad durante la lactancia, considerando el tipo y número de parto de la oveja (Godfrey et al. 1997, Alexandre et al. 2001). No se tiene información sobre el rendimiento y la composición de la canal de corderos de razas de pelo en edad menor de $70 \mathrm{~d}$ (Combellas 1997). Ya que estos estudios se han realizado en etapas posteriores al destete (Partida et al. 2009, Macías-Cruz et al. 2010). Por lo anterior, el objetivo del presente estudio fue evaluar la influencia del tipo de nacimiento de los corderos y el número de parto de la oveja, sobre el crecimiento predestete, rendimiento de la canal, composición corporal de los corderos y el comportamiento productivo de las ovejas.

\section{MATERIALES Y MÉTODOS}

Se estudiaron 25 ovejas Blackbelly $x$ Pelibuey (12 primíparas y 13 multíparas) y sus 39 corderos (20 provenientes de ovejas primíparas y 19 de multíparas). El estudio se llevó a cabo en la unidad ovina experimental del Instituto Nacional de Investigaciones Forestales, Agrícolas y Pecuarias, localizada en el municipio de Huimanguillo, Tabasco, México
(17 50' LN, $93^{\circ} 23^{\prime} \mathrm{LO}$ ), con clima cálido húmedo, lluvias todo el año $(A f)$ y temperatura ambiente media anual de $27.8^{\circ} \mathrm{C}$ (INEGI 2014).

\section{Manejo de la oveja previo al parto y durante la lactancia}

Las ovejas durante los $30 \mathrm{~d}$ previos a la fecha de parto se mantuvieron en pastoreo y se suplementaron con $300 \mathrm{~g}_{\text {oveja }}{ }^{-1} \mathrm{~d}^{-1}$ de un alimento comercial que contenía un $15 \%$ de PC. El nivel de suplementación ofrecido cubrió $30 \%$ de los requerimientos de materia seca (MS) y energía metabolizable (EM) de la etapa de gestación de ovejas Pelibuey (Chávez et al. 1995).

En la primera semana de lactación, las ovejas permanecieron estabuladas con sus camadas y se alimentaron con heno de Cynodon plectostachyus. En tanto que, en la segunda semana de lactación se pastorearon de 8 a $14 \mathrm{~h}$, y el resto del día estuvieron con sus camadas recibiendo heno de $C$. plectostachyus. A partir de la tercera semana y hasta finalizar la etapa de lactación, las ovejas estuvieron en pastoreo de 8 a $18 \mathrm{~h}$, en praderas con C. plectostachyus. El sistema de pastoreo utilizado fue de tipo controlado, la pradera se ocupó durante tres días y se le dio un descanso de $25 \mathrm{~d}$. Durante toda la etapa de lactación las ovejas se suplementaron con $500 \mathrm{~g} \mathrm{oveja}^{-1} \mathrm{~d}^{-1}$ de un alimento comercial con $15 \%$ de PC. El nivel de suplementación ofrecido permitió cubrir $30 \%$ de los requerimientos de MS y EM estimados para la etapa de lactación de ovejas Pelibuey (Chávez et al. 1995).

En las ovejas se determinó el peso y condición corporal (CC) al parto y a los $56 \mathrm{~d}$ posparto. La CC se evaluó utilizando una escala de uno a cinco (Thompson y Meyer 1994). En las ovejas se evaluaron las variables: peso y CC al parto y a los 56 d postparto; cambio de peso durante la lactancia (peso de la oveja al finalizar la lactancia, (kg) - peso de la oveja al parto, $(\mathrm{kg})$ y de CC (CC de la oveja al finalizar la lactancia - CC de la oveja al parto) y cambio de peso diario durante la lactancia como $\frac{\text { pofl en } \mathrm{kg}-\text { pop en } \mathrm{kg}}{56 \mathrm{~d}}$. Donde pofl= peso de la oveja al finalizar la lactancia y $p o p=$ peso de la oveja al parto. De manera simultánea. 
Se registró el peso de la camada al nacimiento y al destete. Este último valor se utilizó para calcular la eficiencia productiva de la oveja con la formula peso de la camada a los $56 \mathrm{~d}$ de edad en $\mathrm{kg}$ (Godfrey et al. 1997).

\section{Manejo de los corderos durante la lactación}

Los corderos estuvieron estabulados toda la etapa de lactación y se alimentaron por amamantamiento controlado, con complemento alimenticio comercial Inicia Corderos con $18 \%$ de PC, que se ofreció a partir del quinto día de edad y heno del pasto $C$. plectostachyus y la leguminosa Gliricidia sepium a libre acceso a partir del día 15 de edad. El acceso al amamantamiento se controló en la segunda semana de vida de los corderos, que consistió en restringir el acceso del cordero al amamantamiento de 8 a $14 \mathrm{~h}$. De la tercera semana hasta concluir el período de la lactancia el acceso se controló de 8 a $18 \mathrm{~h}$.

Para calcular la ganancia diaria de peso (GDP) predestete, los corderos se pesaron al nacimiento y al destete a los $56 \mathrm{~d}$ de edad. Se sacrificaron al destete con un previo ayuno de alimento no lácteo de $14 \mathrm{~h}$. Posteriormente, se retiró la piel, cabeza, extremidades delanteras y traseras a la altura de la articulación carpo-metacarpiana y de la articulación tarso-metatarsiana, vísceras abdominales y torácicas (Martínez et al. 1987). Se registró el peso de la canal caliente y la grasa cavitaria como la grasa interna en las cavidades abdominal y pélvica (Hernández-Espinoza et al. 2012). Posteriormente, la canal se dividió en los cortes cuello, brazo, tórax, abdomen y pierna de acuerdo con Martínez et al. (1987).

El peso vacío fue la diferencia en $\mathrm{kg}$ entre el peso al sacrificio y el peso del contenido digestivo, que se obtuvo por diferencia entre el peso del tracto gastrointestinal (rumen, retículo, omaso, abomaso, intestino delgado e intestino grueso) lleno y vacío. El rendimiento en canal (\%) se de-

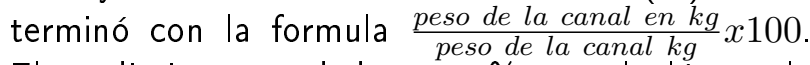
El rendimiento verdadero en \% se calculó con la

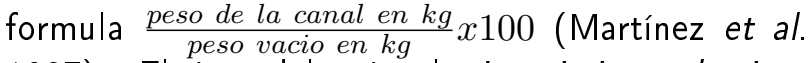
1987). El área del músculo Longissimus dorsi se midió entre la $12^{\mathrm{a}}$ y $13^{\mathrm{a}}$ costilla por medio de una película plástica cuadriculada en $\mathrm{cm}^{2}$ (García et al. 1998). El rendimiento de los cortes primarios en la canal $(\%)$ se determinó a través de la ecuación $\frac{\text { peso del corte primario en } \mathrm{kg}}{\text { peso de la canal en } \mathrm{kg}} x 100$. En los corderos se evaluaron las variables: peso vivo al nacimiento (PN) y al destete (PD); GDP; peso y rendimiento de la canal caliente y verdadero; área del músculo $L$. dorsi; peso y rendimiento de los cortes primarios en la canal: cuello, brazos, tórax, abdomen, piernas y grasa cavitaria.

\section{Diseño experimental y análisis estadístico}

Se utilizó un diseño factorial de dos factores; que fueron el tipo de nacimiento (TN) de los corderos (sencillo y múltiple) y el número de parto (NP) de las ovejas (primípara y multípara). En el grupo con nacimiento múltiple se incluyeron los datos de una oveja que al parto y al destete tuvo tres corderos. En el estudio del crecimiento del cordero, la unidad experimental fue el cordero, mientras que en el estudio de la eficiencia productiva de la oveja, la unidad experimental fue la oveja o la camada. Todos los análisis se efectuaron con el paquete estadístico SAS 9.0 (SAS 2002) y se probó su normalidad con la prueba de Shapiro-Wilk's. Los análisis se efectuaron con el procedimiento GLM. En los modelos finales se excluyó la interacción $\mathrm{NP} \times \mathrm{TN}$, cuando no fue significativa $(\mathrm{p}<0.05)$, mientras que los datos de CC y de variables que no mostraron normalidad se analizaron con la prueba de la suma de rangos de Wilcoxon para datos no pareados (Milton 2001).

\section{RESULTADOS}

\section{Crecimiento predestete}

La interacción NP x TN no afectó ( $p>0.05)$ el PN, PD y la GDP predestete de los corderos. El NP de la oveja solo afectó $(p<0.05)$ el PN de los corderos. Los corderos provenientes de ovejas primíparas tuvieron un menor PN con respecto al de corderos de ovejas multíparas, con peso de 2.6 \pm 0.14 y $3.1 \pm 0.14 \mathrm{~kg}$, respectivamente.

Con excepción del PN, el TN influyó $(p<$ 
$0.05)$ en el PD y GDP de los corderos. Las medias de cuadrados mínimos \pm error estándar (EE) para PN, PD y GDP fueron de $2.9 \pm 0.17 \mathrm{~kg}, 11.8 \pm$ $0.56 \mathrm{~kg}$ y $159 \pm 8 \mathrm{~g}$ en corderos de nacimiento sencillo y de $2.7 \pm 0.11 \mathrm{~kg}, 10.3 \pm 0.37 \mathrm{~kg}$ y 136 $\pm 5 \mathrm{~g}$ en corderos de nacimiento múltiple, respectivamente.

\section{Producción de la canal}

El NP influyó $(\mathrm{p}<0.05)$ sobre el área del $L$. dorsi. Los corderos provenientes de ovejas primíparas tuvieron una menor área del $L$. dorsi con respecto al de corderos de multíparas, con $6.9 \pm$ 0.50 y $8.6 \pm 0.45 \mathrm{~cm}^{2}$, respectivamente. En el resto de las variables no se detectó $(p>0.05)$ influencia del NP.

Con excepción rendimiento de la canal, el resto de las variables relacionadas con el estudio de la canal de los corderos fueron afectadas $(p<0.05)$ por el TN. Los corderos de nacimiento sencillo tuvieron mayor peso y rendimiento de la canal, peso de los cortes primarios, peso de la grasa cavitaria y área del músculo $L$. dorsi con respecto a corderos de nacimiento múltiple (Tabla 1 ).

Con excepción del rendimiento del tórax $(\mathrm{p}<$ 0.05 ), el resto de las variables relacionadas con el estudio de la canal de los corderos no fueron afectadas por la interacción NP $\times$ TN $(p>0.05)$. Las medias de cuadrados mínimos \pm EE para rendimiento del tórax en la canal en primíparas con parto sencillo, primíparas con parto múltiple, multíparas con parto sencillo y multíparas con parto múltiple fueron de $26.4 \pm 0.85,23.7 \pm 0.49,25.4 \pm 0.71$, y $26.0 \pm$ $0.55 \%$, respectivamente. Las medias generales \pm desviación estándar de los rendimientos de los cortes en la canal fueron de $8.3 \pm 1.4$ en cuello, $19.5 \pm$ 1.1 en brazo, $25.1 \pm 2.1$ en tórax, $19.9 \pm 2.3$ en abdomen y $25.9 \pm 2.1 \%$ en pierna.

\section{Productividad de las ovejas}

La interacción NP x TP no afectó ( $p>0.05)$ las variables evaluadas en las ovejas y sus camadas. EI NP tuvo influencia $(\mathrm{p}<0.05)$ sobre las variables: peso al parto, cambio de peso, cambio de peso diario y CC al parto. No obstante, el NP no afectó ( $p>$
$0.05)$ al resto de las variables evaluadas en las ovejas y su camada (Tabla 2). Las variables peso vivo a los 56 d posparto, $C C$ al parto y en el día 56 posparto y cambio en la CC no fueron afectadas ( $p>0.05)$ por el TP. Sin embargo, el TN influyó $(p<0.05)$ en el resto de las variables incluidas en el estudio de las ovejas y su camada (Tabla 2).

\section{DISCUSIÓN}

\section{Crecimiento predestete}

El crecimiento prenatal, expresado como el PN fue afectado por la interacción entre el feto y el ambiente materno. El PN de corderos provenientes de madres multíparas fue mayor que el de corderos de madres primíparas, lo que coincide con Carrillo et al. (1987) y Ríos-Utrera et al. (2014), quienes reportan que en ovejas de pelo, el PN fue mayor en corderos nacidos de ovejas multíparas que en corderos de ovejas primíparas. Probablemente el útero de las ovejas multíparas tenga mayor volumen que el de las primíparas, al respecto se sabe que un mayor volumen del útero favorece el crecimiento y desarrollo fetal (Gootwine et al. 2007), lo que puede explicar la superioridad en PN de los corderos de ovejas multíparas. También, se ha documentado que factores ambientales como, la edad, talla y número de parto, influyen en el crecimiento fetal (Jainuden y Hafez 1996, Lawrence y Fowler 2002).

Un factor importante que determina el crecimiento fetal, es el número de fetos presentes en la hembra. Se ha reportado que en ovinos, el PN del cordero disminuye con el incremento del número de corderos nacidos (Gootwine 2005). Sin embargo, en el presente estudio el TN no afectó el PN de los corderos, pesando corderos de nacimiento múltiple $94 \%$ del PN de los de nacimiento sencillo. Un resultado similar fue reportado para ovejas Pelibuey, en el que se encontró que los corderos gemelos pesaron $96 \%$ del PN de corderos de nacimiento sencillo (Macías-Cruz et al. 2009). Mientras que otros estudios, han encontrado que el TN afecta el PN de los corderos, pesando los corderos gemelos entre 82 y $88 \%$ del PN de los corderos de nacimiento sencillo (Hinojosa-Cuéllar et al. 2012, Ríos-Utrera 
Tabla 1. Influencia del tipo de nacimiento sobre la composición corporal de corderos Blackbelly $\mathrm{x}$ Pelibuey.

\begin{tabular}{lrr}
\hline Variable & \multicolumn{2}{c}{ Tipo de nacimiento } \\
\cline { 2 - 3 } & Sencillo & Múltiple \\
\hline Edad al sacrificio (d) & 56 & 56 \\
Peso al sacrificio (kg) & $11.8 \pm 0.56^{a}$ & $10.3 \pm 0.37^{b}$ \\
Peso vacío (kg) & $10.5 \pm 0.49^{a}$ & $9.0 \pm 0.33^{b}$ \\
Peso de la canal (kg) & $5.7 \pm 0.28^{a}$ & $4.8 \pm 0.19^{b}$ \\
Rendimiento de la canal (\%) & $47.9 \pm 0.60^{a}$ & $46.1 \pm 0.40^{b}$ \\
Rendimiento verdadero de la canal (\%) & $54.2 \pm 0.54$ & $52.9 \pm 0.36$ \\
Cuello (g) & $465 \pm 25^{a}$ & $390 \pm 17^{b}$ \\
Brazo (g) & $1081 \pm 56^{a}$ & $937 \pm 38^{b}$ \\
Pierna (g) & $1439 \pm 75^{a}$ & $1239 \pm 50^{b}$ \\
Tórax (g) & $1475 \pm 87^{a}$ & $1187 \pm 58^{b}$ \\
Abdomen (g) & $1149 \pm 70^{a}$ & $952 \pm 47^{b}$ \\
Área del músculo Longissimus dorsi (cm $\left.{ }^{2}\right)$ & $8.6 \pm 0.56^{a}$ & $7.1 \pm 0.37^{b}$ \\
Grasa cavitaria (g) & $234 \pm 29^{a}$ & $133 \pm 21^{b}$ \\
\hline$a, b$ medias de cuadrados mínimos \pm error estándar con diferente superíndice \\
dentro de la misma fila indican diferencia significativa ( $\mathrm{p}<0.05)$.
\end{tabular}

Tabla 2. Influencia del número y tipo de parto sobre la eficiencia productiva de ovejas Blackbelly $\mathrm{x}$ Pelibuey.

\begin{tabular}{lcccc}
\hline Variable & \multicolumn{2}{c}{ Número de parto } & \multicolumn{2}{c}{ Tipo de parto } \\
& Primípara & Multípara & Sencillo & Múltiple \\
\hline Peso al parto (kg) & $32.0 \pm 1.62 \mathrm{a}$ & $37.6 \pm 1.54^{b}$ & $31.9 \pm 1.62^{a}$ & $37.7 \pm 1.54^{b}$ \\
Peso al día 56 postparto (kg) & $30.5 \pm 1.16$ & $32.9 \pm 1.11$ & $30.0 \pm 1.16$ & $33.4 \pm 1.11$ \\
Cambio de peso diario (g) & $-27 \pm 13^{a}$ & $-84 \pm 13^{b}$ & $-33 \pm 13^{a}$ & $-77 \pm 13^{b}$ \\
Cambio de peso (kg) & $-1.5 \pm 0.75^{a}$ & $-4.7 \pm 0.71^{b}$ & $-1.9 \pm 0.75^{a}$ & $-4.3 \pm 0.71^{b}$ \\
Condición corporal al parto & $3.8 \pm 0.11^{a}$ & $3.2 \pm 0.19^{b}$ & $3.5 \pm 0.19$ & $3.5 \pm 0.18$ \\
Condición corporal al día 56 postparto & $2.7 \pm 0.14$ & $2.4 \pm 0.20$ & $2.6 \pm 0.15$ & $2.5 \pm 0.22$ \\
Cambio de condición corporal & $-1.1 \pm 0.16$ & $-0.8 \pm 0.22$ & $-0.9 \pm 0.19$ & $-1.0 \pm 0.21$ \\
Peso de la camada al nacimiento (kg) & $4.0 \pm 0.29$ & $4.5 \pm 0.28$ & $2.9 \pm 0.29^{a}$ & $5.7 \pm 0.28^{b}$ \\
Peso de la camada al destete (kg) & $16.3 \pm 1.01$ & $16.9 \pm 0.96$ & $11.9 \pm 1.01^{a}$ & $21.4 \pm 0.96^{b}$ \\
Productividad & $0.52 \pm 0.02$ & $0.51 \pm 0.02$ & $0.39 \pm 0.02^{a}$ & $0.64 \pm 0.02^{b}$ \\
\hline a,b medias de cuadrados mínimos \pm error estándar con diferente superíndice dentro de la misma fila y \\
mismo efecto principal indica diferencia significativa (p < 0.05 ).
\end{tabular}

et al. 2014). En relación a la ausencia de influencia del TN sobre el PN de los corderos, al respecto Gootwine (2005) encontró que el PN se reduce con el incremento del tamaño de la camada. Mientras que Greenwood et al. (2000) reportan asociación positiva entre el peso de la oveja al apareamiento con el peso de la placenta. En el presente estudio, las ovejas con un tipo de parto múltiple, mostraron un mayor peso al parto con relación a las hembras de parto sencillo, por lo que es probable que la placenta de las ovejas con parto múltiple tenga mayor peso y eficiencia que la placenta de las ovejas con parto sencillo, lo que puede explicar la ausencia de diferencias en el PN.

EI PD y GDP de los corderos de nacimiento múltiple representó 91 y $87 \%$ de el de los corderos de nacimiento sencillo. Lo que coincide con Rajab et al. (1992), Hinojosa-Cuéllar et al. (2009) y Ríos-Utrera et al. (2014) quienes reportan que, el PD y GDP predestete están influenciados por el TN, siendo superiores los valores en corderos de nacimiento sencillo que los de nacimiento múltiple. Al respecto se sabe que la frecuencia de amamantamiento de los corderos de nacimiento gemelar es mayor que la de corderos de nacimiento sencillo (Teke y Akdag 2012) y que la producción de leche en ovejas con dos corderos es mayor que la de ovejas con un cordero (Snowder y Glimp 1991). Sin embargo, una mayor frecuencia de amamantamiento y mayor producción láctea no son suficientes para que los corderos de nacimiento gemelar logren un PD y GDP predestete similar a la de corderos de 
nacimiento sencillo.

El crecimiento predestete es afectado por el nivel de nutrición materna y por factores que influyen en la producción de leche (Bell et al. 1987). En el presente estudio, las ovejas con parto múltiple y sencillo recibieron el mismo manejo nutricional, situación que pudo poner en desventaja a las ovejas con parto múltiple, debido a su mayor demanda de nutrimentos por su mayor peso vivo y por amamantar a dos o tres crías (NRC 2007). Lo que ocasionó menor eficiencia de crecimiento de los corderos de nacimiento múltiple, ya que la leche disponible se tiene que compartir entre el número de crías, y por consiguiente menor GDP.

\section{Producción de la canal}

El peso vivo de un animal no siempre tiene correlación con la cantidad de productos vendibles (Owens et al. 1993). De ahí la importancia de identificar los factores que afectan el rendimiento y composición de la canal. Se ha reportado que el TN afecta el peso de la canal de los ovinos de lana en finalización, teniendo los ovinos de nacimiento gemelar $90 \%$ del peso de la canal de los corderos de nacimiento sencillo (McCoard et al. 2015). En el presente estudio, el peso de la canal de los corderos de nacimiento múltiple fue $86 \%$ del peso de la canal de los corderos de nacimiento sencillo. Es posible, que las diferencias en el peso de la canal de corderos de nacimiento sencillo y múltiple se deba al peso de la masa muscular y el consumo individual de leche. Ya que el peso de la masa muscular, está en función del número y tamaño de las fibras musculares. En los fetos gemelos, se ha detectado que estos tienen un menor peso de su masa muscular y concentración de aminoácidos en el músculo semitendinosus, al compararlos con fetos de gestación sencilla (McCoard et al. 2000, Sales et al. 2013). La importancia de los aminoácidos radica en que estos están implicados en las vías que regulan la síntesis de proteínas (Wu 2009). El menor peso de la masa muscular de los corderos gemelos al nacimiento, puede explicar el retardo en su desarrollo muscular posnatal. También, se ha reportado que el nivel de producción de leche de las ovejas que amamantan dos crías puede ser mayor (Ramsey et al. 1998, Jawasreh y Khasawneh 2007) o similar al de ovejas con una sola cría (Godfrey et al. 1997). Sin embargo, en el caso de corderos gemelos, se debe compartir la cantidad de leche que produce la oveja, lo que limita el nivel de consumo de nutrimentos. El rendimiento de la canal fue afectado por el TN, la canal de corderos de nacimiento múltiple tuvo $96 \%$ de la canal de corderos de nacimiento sencillo. Pero al considerar el peso vacío del cordero en el rendimiento verdadero de la canal, no se detectaron diferencias. Lo que indica que a una misma edad, el rendimiento verdadero de la canal se mantiene cuando las diferencias en el peso vacío de los corderos son de baja magnitud.

El rendimiento de la canal de los corderos evaluados fue ligeramente inferior al reportado en corderos de pelo de la raza West African sacrificados a los $70 \mathrm{~d}$ de edad y con mayor peso (Combellas 1997) y al de corderos de lana de la raza Suffolk Down sacrificados a los $23 \mathrm{~d}$ de edad y con menor peso (Pérez et al. 2002). Sin embargo, el rendimiento verdadero de la canal de los corderos fue similar al encontrado en corderos West African y Suffolk Down (Combellas 1997). Las diferencias entre estos estudios se deben al rendimiento comercial de la canal, raza, edad y tipo de nacimiento, ya que en rendimiento verdadero de la canal fueron similares.

En los corderos de nacimiento múltiple el peso de los cortes primarios representó entre 80.4 y $86.7 \%$ del peso de los de nacimiento sencillo. El mayor peso de los cortes primarios en corderos de nacimiento sencillo coincide con lo reportado en corderos de lana sacrificados a 73 y $110 \mathrm{~d}$, en los que el peso de la pierna fue mayor en los de nacimiento sencillo que en los de nacimiento gemelar (McCoard et al. 2010). Específicamente, el peso de la pierna de los corderos de nacimiento múltiple representó 86 $\%$ del peso de la pierna de los de nacimiento sencillo. Al parecer, los corderos de nacimiento sencillo tuvieron una ventaja inicial sobre los de nacimiento múltiple en la composición de la masa muscular (McCoard et al. 2000, Sales et al. 2013) y en el consumo individual de leche (Godfrey et al. 1997, 
Ramsey et al. 1998, Jawasreh y Khasawneh 2007). Esta ventaja se mantuvo hasta el destete y se detectó en el mayor peso de la canal y en el peso de los cortes primarios objeto de la investigación.

El área del músculo $L$. dorsi en corderos provenientes de ovejas primíparas fue $80 \%$ del área de corderos de multíparas. No hay estudios sobre la influencia del NP de las ovejas, sobre la variación del área del $L$. dorsi de los ovinos en edades menores o mayores a $90 \mathrm{~d}$. Si se considera que el número de corderos al destete fue similar entre ovejas primíparas $(1.5 \pm 0.06)$ y multíparas $(1.6 \pm 0.06)$, es probable que los corderos de ovejas multíparas hayan consumido mayor cantidad de leche con respecto a los corderos de primíparas. Un mayor consumo de nutrimentos puede favorecer un crecimiento diferenciado en algunas regiones de la canal. Al respecto, existen evidencias que indican que la producción de leche en ovejas Awassi se incrementó de forma gradual después del primer parto (Jawasreh y Khasawneh 2007). Mientras que Partida y Martínez (2010) indican que el crecimiento de las regiones corporales y composición tisular en la canal ovina es afectado por la concentración energética en la dieta. Por lo que es probable que la mayor área del músculo $L$. dorsi en corderos provenientes de ovejas multíparas se deba a un mayor consumo de nutrimentos con respecto al de corderos de primíparas.

Los corderos de nacimiento múltiple tuvieron $82.6 \%$ del área del músculo $L$. dorsi de los de nacimiento sencillo, lo que no coincide con Jucá et al. (2014), quienes encontraron que el TN no afectó el área del músculo $L$. dorsi en corderos de la raza Santa Ines a los 112 d. Mientras que Sales et al. (2013) señalan que en los fetos gemelos, el peso del músculo semitendinosus fue $17 \%$ menor que el de los fetos provenientes de gestación sencilla. Una situación similar pudiera presentarse en el $L$. dorsi de los corderos de nacimiento múltiple y mantenerse durante la etapa posnatal, lo que explicaría las diferencias en el área del $L$. dorsi entre corderos de nacimiento múltiple y sencillo.

El crecimiento incluye el depósito de grasa corporal, aunque la masa muscular es la de interés primario en la producción de carne. Particularmente, el depósito de grasa perineal precede el depósito intermuscular, subcutáneo e intramuscular (Owens et al. 1993). El incremento en la tasa de depósito de grasa en los animales sin destetar, muestra una relación directa con el suministro de ácidos grasos de cadena larga preformados provenientes de la leche, que se caracteriza por poseer un alto contenido de grasa (Castellanos y Valencia 1982, Bell et al. 1987, Martínez-González et al. 2015). Los corderos de nacimiento múltiple tuvieron $61 \%$ de la grasa cavitaria encontrada en los de nacimiento sencillo. Esta diferencia puede ser atribuida a un menor consumo de leche, y por consiguiente de grasa, en los corderos de nacimiento múltiple con respecto a los de nacimiento sencillo. En estudios futuros se deberá estudiar la influencia del tipo de nacimiento en el desarrollo metabólico y endocrino, debido a que en la etapa de crecimiento prenatal la concentración sanguínea de glucosa y aminoácidos adquieren relevancia en el metabolismo, mientras que en la etapa de crecimiento posnatal la dieta que reciben contiene un alto contenido de grasa y una menor proporción de carbohidratos (Greenwood et al. 2002, Martínez-González et al. 2015).

En corderos Suffolk Down (Pérez et al. 2002) y Segureña (Peña et al. 2005) sacrificados entre los 10 y $13 \mathrm{~kg}$ de peso vivo, los cortes primarios que tuvieron la mayor y menor proporción de la canal fueron la pierna y el cuello, lo que coincide con lo encontrado en el presente estudio. No obstante, el rendimiento de la pierna fue de menor magnitud. Esta diferencia puede ser atribuida a una mayor ganancia de peso predestete en los corderos de razas especializadas en la producción de carne, con respecto a los de razas de pelo. Al respecto, se ha reportado que los corderos Pelibuey tienen 66.9 $\%$ de la ganancia de peso predestete que los corderos Dorper (Hinojosa-Cuéllar et al. 2009).

En el resto de los cortes, no se realizaron comparaciones de rendimiento debido a diferencias en la definición y número de secciones corporales que comprenden los cortes primarios utilizados en los estudios que describen la composición de la canal de 
corderos (Peña et al. 2005, Luaces et al. 2008, Partida y Braña 2011).

En ovinos Blackbelly $x$ Pelibuey y Pelibuey sacrificados con mayor peso al del presente estudio, se ha encontrado que el corte primario con mayor proporción en la canal es el tórax, seguido de la pierna, brazo, abdomen y cuello (Cantón et al. 1992, Partida y Martínez 2010), lo que no coincide con lo encontrado en el presente estudio. La diferencia puede atribuirse a la edad, TN, peso, raza y tipo de alimentación (Martínez et al. 1990, Luaces et al. 2007, Pérez et al. 2012). Al respecto, se sabe que el peso al sacrificio y el nivel energético de la dieta no afecta la proporción de los cortes primarios de la canal de ovinos Pelibuey finalizados (Partida y Martínez 2010). Sin embargo, es posible que en corderos con edad menor a $60 \mathrm{~d}$, la proporción de los cortes primarios de la canal difiera a la de ovinos en finalización, en su orden de participación debido a que los cortes primarios de la canal tienen una velocidad de crecimiento diferente, la que está en función de la composición de hueso, músculo y grasa (Lawrence y Fowler 2002).

El rendimiento del tórax fue afectado por la interacción NP $\times$ TN. En los corderos de nacimiento múltiple provenientes de ovejas primíparas, se encontró que el tórax tuvo menor proporción en la canal con respecto a los corderos de nacimiento sencillo provenientes de primíparas y de nacimiento múltiple de ovejas multíparas. Al respecto, Luaces et al. (2007) indican que la madurez de los cortes de la canal en corderos de la raza Gallega difieren y el corte costillar tuvo madurez tardía. También, Jucá et al. (2014) encontraron que los corderos de nacimiento trillizo tuvieron un menor perímetro torácico que los de nacimiento gemelar y sencillo. Una maduración tardía de la región tórax y menor perímetro torácico pueden explicar la menor proporción del tórax en la canal en corderos de nacimiento múltiple de ovejas primíparas.

\section{Productividad de las ovejas}

Las ovejas multíparas tuvieron $118 \%$ del peso al parto de las primíparas. Lo que coincide con lo reportado por Rajab et al. (1992), quiénes en- contraron que en ovejas de las razas Morada Nova, Santa Inés y Somalí el peso al parto se incrementa con la edad. Mientras que Molina et al. (1991) reportan que las ovejas Manchega con edad mayor a tres años son más pesadas al parto que las ovejas con menor edad. La diferencia en el peso al parto entre ovejas primíparas y multíparas se relaciona con el peso a la primera concepción y el tipo de alimentación (Owens et al. 1993). Las ovejas multíparas perdieron el triple de peso durante la lactancia con respecto a las primíparas. Lo que se puede explicar, por el mayor peso vivo de las ovejas multíparas, lo que implica mayor demanda de nutrimentos para cubrir los requerimientos de mantenimiento (NRC 2007). Bajo similar manejo nutricional y capacidad productiva entre ovejas primíparas y multíparas, en las ovejas multíparas se espera un mayor uso del tejido adiposo y masa muscular para sustentar los requerimientos de nutrimentos de la producción de leche (Chávez et al. 1995).

En lo que respecta a la CC, las ovejas multíparas tuvieron un menor valor al parto con respecto a las primíparas, esta menor CC puede atribuirse a que las ovejas presentan lactancia previa, en donde es frecuente que ocurra una reducción de peso vivo y CC, cuando son alimentadas con base en el pastoreo (Godfrey et al. 1997). Es probable que durante el período de gestación de las ovejas multíparas, el consumo de nutrimentos aportados por el consumo voluntario de pasto durante el pastoreo y el complemento alimenticio asignado, un mes previo a la fecha de parto, no fue suficiente para que alcanzaran una CC similar a las primíparas.

El NP no afectó el cambio de CC, esto coincide con lo reportado en ovejas Manchegas, en donde el cambio de CC durante la lactancia fue negativo y de la misma magnitud entre ovejas con menos y más de tres años (Molina et al. 1991). La CC es una variable de fácil y de rápida medición, pero subjetiva. Sin embargo, la puntuación de CC en ovejas adultas se asocia con el contenido de lípidos en la canal, además de que es un buen indicador de las reservas de energía disponibles en las ovejas (Sanson et al. 1993). Es posible que la magnitud de los cambios detectados en la CC en $56 \mathrm{~d}$, no sean 
suficientes para detectar diferencias significativas en su cambio entre ovejas primíparas y multíparas. En este sentido, no se encontraron estudios en ovejas en lactación que muestren la relación entre una unidad de cambio en la CC con el peso vivo. Sin embargo, en ovejas Pelibuey no lactantes y no gestantes se ha establecido que una unidad de cambio en la CC corresponde a $5.8 \mathrm{~kg}$ de peso vivo (Chay-Canul et al. 2011). En este estudio, las ovejas primíparas y multíparas perdieron entre 1.5 y $4.7 \mathrm{~kg}$, situación que pudiera explicar que la reducción en la CC no haya sido mayor a una unidad de CC.

El peso de la camada al nacimiento y al destete no fue afectado por el NP, esto coincide parcialmente con lo indicado por Cadenas-Cruz et al. (2012) quienes encontraron que ovejas Blackbelly con uno y dos partos, tienen un peso de camada al nacimiento similar, pero, menor al de ovejas con más de dos partos. Mientras que en el caso del peso de la camada al destete, Hinojosa-Cuéllar et al. (2015) indican que las ovejas de pelo en sus primeros cuatro partos producen camadas al destete con peso similar. En contraste respecto a ovejas Blackbelly (Cadenas-Cruz et al. 2012) y Pelibuey (MagañaMonforte et al. 2013) con más de dos partos, que producen camadas más pesadas al destete que las de primer parto.

La productividad de las ovejas fue similar entre primíparas y multíparas. Sin embargo, los valores obtenidos fueron superiores a los reportados para ovejas Blackbelly, Florida y St. Croix (Godfrey et al. 1997). Las ovejas con tipo de parto múltiple, tuvieron un mayor peso al parto que las ovejas con parto sencillo, un resultado similar, pero de menor magnitud fue reportado en ovejas Morada Nova, Santa Inés y Somalí (Rajab et al. 1992).

El TP afectó el cambio de peso y el cambio de peso diario de las ovejas. Las ovejas con parto múltiple perdieron más peso durante la lactancia que las ovejas de parto sencillo. Las diferencias en el cambio de peso entre ovejas con parto múltiple y parto sencillo pueden atribuirse a que las ovejas de parto múltiple tienen mayores requerimientos de nutrimentos, que las ovejas de parto sencillo (NRC 2007). Las ovejas de parto múltiple y sencillo es- tuvieron expuestas a un similar manejo nutricional, esta situación probablemente favoreció que las ovejas de parto múltiple perdieran más peso debido a un mayor uso de sus reservas corporales para apoyar la mayor producción de leche (Alexandre et al. 2001).

Las ovejas con parto sencillo y múltiple, perdieron una unidad de CC durante la lactancia, sin que se detectara influencia del TP. Este resultado difiere de lo indicado por Alexandre et al. (2001), quienes al ofrecer un alto nivel de complementación alimenticia a ovejas de pelo, permite que las ovejas con parto sencillo muestren un incremento de una unidad de CC durante la lactancia. Mientras que las ovejas con parto gemelar, muestran un incremento en su CC de 0.2 unidades. La reducción en CC detectada tanto en ovejas con parto múltiple como en las de parto sencillo, sugiere que estas no lograron cubrir sus requerimientos de nutrimentos para la etapa de lactación (NRC 2007). Las ovejas de parto múltiple tuvieron mayor productividad con respecto a las de parto sencillo, debido al mayor peso de la camada al nacimiento y al destete. La ventaja de las ovejas de parto múltiple sobre las de parto sencillo, se atribuye al mayor número de corderos en la camada. Lo que coincide con lo reportado en ovejas Morada Nova, Santa Inés y Somalí en las que la camada formada por dos corderos tienen un peso al nacimiento y al destete del 156 y $149 \%$, respectivamente, del peso de camadas con un solo cordero (Rajab et al. 1992). Al respecto, Hinojosa-Cuéllar et al. (2015) encontraron que el TP influye sobre el peso de la camada al destete, siendo más pesadas las camadas con dos corderos que las que tienen uno. En lo referente a la eficiencia de producción, Godfrey et al. (1997) reportan que las ovejas con parto gemelar tienen una mayor productividad que la presentada en ovejas con parto sencillo, lo que coincide con lo encontrado en el presente estudio.

\section{CONCLUSIONES}

El tipo de parto afectó la ganancia diaria de peso predestete, peso al sacrifico y rendimiento de la canal caliente de los corderos Blackbelly x Pelibuey. 
Los corderos de nacimiento sencillo tuvieron un comportamiento productivo superior al de corderos de nacimiento múltiple. El número de parto de las ovejas influyó en el peso al nacimiento y área del músculo Longissimus dorsi de los corderos, los corderos provenientes de ovejas multíparas tuvieron mayor peso al nacimiento y área del músculo $L$. dorsi con respecto a los corderos de ovejas primíparas. EI número de parto y el tipo de parto afectaron el cambio de peso de las ovejas, las ovejas multíparas y ovejas con parto múltiple tuvieron mayor pérdida de peso durante la etapa de lactancia, que las ovejas primíparas y ovejas con parto sencillo. Las ovejas con parto múltiple mostraron mayor eficiencia productiva que las de parto sencillo.

\section{AGRADECIMIENTOS}

Se agradece al CONACYT la beca otorgada al primer autor para realizar sus estudios de Maestría en Ciencias dentro del programa Producción Agroalimentaria en el Trópico del Colegio de Postgraduados Campus Tabasco.

\section{LITERATURA CITADA}

Alexandre G, Archimède H, Chevaux E, Aumont G, Xandè (2001) Feeding supply of suckling Martinik ewes reared in intensive conditions: effects of supplement levels and litter size. Animal Research 50: 213-221.

Bell AW, Bauman DE, Currie WB (1987) Regulation of nutriente partitioning and metabolism during preand postnatal growth. Journal of Animal Science 65 (Suppl. 2): 186-212.

Cadenas-Cruz PJ, Oliva-Hernández J, Hinojosa-Cuéllar JA (2012) Productivity of Blackbelly ewes and their hybrid litter under grazing Journal of Animal Veterinary Advances 11: 97-102.

Cantón JG, Velázquez A, Castellanos A (1992) Body composition of pure and crossbred Blackbelly sheep. Small Ruminant Research 7: 61-66.

Carrillo L, Velázquez A, Ornelas T (1987) Algunos factores ambientales que afectan el peso al nacer y al destete de corderos Pelibuey. Técnica Pecuaria en México 25: 289-295.

Castellanos A, Valencia M (1982) Estudio cuantitativo y cualitativo de la producción láctea de la borrega Pelibuey. Producción Animal Tropical 7: 245-255.

Chávez G, Castellanos A, Velázquez PA (1995) Producción de las ovejas Pelibuey pre y postparto alimentadas con diferentes aportes nutricionales. Técnica Pecuaria en México 33: 183-195.

Chay-Canul AJ, Ayala-Burgos AJ, Kú-Vera JC, Magaña-Monforte JG, Ferrell Cl (2011) Metabolizable energy intake and changes in body weight and body condition of Pelibuey ewes fed three levels of roughage diets under tropical conditions. Tropical and Subtropical Agroecosystems 14: 777-786.

Combellas JB De (1997) Calidad de la canal en ovejas West-African y sus cruces. En: González-Stagnaro C (compilador) Ovinos de pelo. Ovis. Tratado de Patología y Producción ovina. Luzan 5, SA de Ediciones Madrid. España. pp: 75-82.

García JA, Nuñez FA, Rodríguez FA, Prieto C, Molina NI (1998) Calidad de la canal y de la carne de borregos Pelibuey castrados. Técnica Pecuaria en México 36: 225-232.

Godfrey RW, Gray ML, Collins JR (1997) Lamb growth and milk production of hair and wool sheep in a semi-arid tropical environment. Small Ruminant Research 24: 77-83.

Goodtwine E (2005) Variability in the rate of decline in birth weight as litter size increases in sheep. Animal Science 81: 393-398. 
Goodtwine E, Spencer TE, Bazer FW (2007) Litter-size-dependent intrauterine growth restriction in sheep. Animal 1: 547-564.

Greenwood PL, Slepetis RM, Bell AW (2000) Influences on fetal and placental weights during mid to late gestation in prolific ewes well nourished throughout pregnancy. Reproduction, Fertility and Development 12: $149-156$.

Greenwood PL, Hunt AS, Slepetis RM, Finnerty KD, Alston C, Beermann DH et al. (2002) Effects of birth weight and postnatal nutrition on neonatal sheep: III. Regulation of energy metabolism. Journal of Animal Science 80: 2850-2861.

Hernández-Espinoza DF, Oliva-Hernández J, Pascual-Córdova A, Hinojosa-Cuéllar JA (2012) Descripción de medidas corporales y composición de la canal en corderas Pelibuey: Estudio preliminar. Revista Científica FCV-LUZ XXII: 24-31.

Hinojosa-Cuéllar JA, Regalado-Arrázola F M, Oliva-Hernández J (2009) Crecimiento prenatal y predestete en corderos Pelibuey, Dorper, Katahdin y sus cruces en el sureste de México. Revista Científica FCV-LUZ XIX: 522-532.

Hinojosa-Cuéllar JA, Oliva-Hernández J, Torres-Hernández G, Segura-Correa JC, Aranda-Ibáñez EM, GonzálezCamacho JM (2012) Factores que afectan el crecimiento predestete de corderos Pelibuey en el trópico húmedo de México. Universidad y Ciencia 28: 163-171.

Hinojosa-Cuéllar JA, Oliva-Hernández J, Torres-Hernández G, Segura-Correa JC, González-Garduño R (2015) Productividad de ovejas F1 Pelibuey x Blackbelly y sus cruces con Dorper y Katahdin en un sistema de producción del trópico húmedo de Tabasco, México. Archivos de Medicina Veterinaria 47: 167-174.

INEGI (2014) Anuario estadístico y geográfico de Tabasco 2014. INEGI, México. 414p.

Jainudeen MR, Hafez ESE (1996) Estación, fisiología prenatal y parto. En: Hafez ESE (ed). Reproducción e inseminación artificial en animales. $6^{t a}$ Edición. McGraw-Hill Interamericana. México. pp: 203-224.

Jawasreh KIZ, Khasawneh AZ (2007) Genetic evaluation of milk production traits i awassi sheep in Jordan. Egyptian Journal odf Sheep and Goat Sciences 21: 83-100.

Jucá AF, Faveri CJ, Melo-Filho GM, Ribeiro-Filho AL, Azevedo HC, Muniz EN et al. (2014) Performance of the Santa Ines breed raised on pasture in semiarid tropical regions and factors that explain trait variation. Tropical Animal Health Production 46: 1249-1256.

Lawrence TLJ, Fowler VR (2002) Growth of farm animals. CABI Publishing. UK. 347p.

Luaces ML, Calvo C, Fernández B, Viana JL, Fernández B, Sánchez L (2007) Estudio de las pieza comerciales y su desarrollo en canales de corderos de la raza ovina Gallega. Archivos de Zooteccnia 56: 157-168.

Luaces ML, Calvo C, Fernández B, Viana JL, Sánchez L (2008) Ecuaciones predictoras de la composición tisular de las canales de corderos de raza Gallega. Archivos de Zootecnia 57: 3-14.

Macías-Cruz U, Álvarez-Valenzuela FD, Correa-Calderón A, Molina-Ramírez L, Gónzalez-Reyna A, SotoNavarro $S$ et al. (2009) Pelibuey ewe productivity and subsequent pre-weaning lamb performance using hair-sheep breeds under a confinement system. Journal of Applied Animal Research 36: 255-260.

Macías-Cruz U, Álvarez-Valenzuela FD, Rodríguez-García J, Correa-Calderón A, Torrentera-Olivera NG, Molina-Ramírez L et al. (2010) Crecimiento y características de canal en corderos Pelibuey puros y cruzados F1 con razas Dorper y Katahdin en confinamiento. Archivos de Medicina Veterinaria 42: 147-154. 
Magaña-Monforte JG, Huchin-Cab M, Ake-López RJ, Segura-Correa JC (2013) A field study of reproductive performance and productivity of Pelibuey ewes in Southeastern Mexico. Tropical Animal Health and Production 45: 1771-1776.

Martínez AMM, Bores RF, Castellanos AF (1987) Zoometría y predicción de la composición corporal de la borrega Pelibuey. Técnica Pecuaria en México 25: 72-84.

Martínez AMM, Bores R, Velázquez PA, Castellanos AF (1990) Influencia de la castración y del nivel energético de la dieta sobre el crecimiento y composición corporal del borrego Pelibuey. Técnica Pecuaria en México 28: 125-132.

Martínez-González S, Escalera-Valente F, Gómez-Dánes AA, Plascencia A, Loya-Olguin JL, Ramírez-Ramírez JC, et al. (2015) Influence of levels of DL-malic acid supplementation on milk production and composition in lactating Pelibuey ewes and pre-weaning weight gain of their suckling kids. Journal of Applied Animal Research 43: 92-96.

McCoard SA, McNabb WC, Peterson SW, McCutcheon SN, Harris PM (2000) Muscle growth, cell number, type and morphometry in single and twin fetal lambs during mid to late gestation. Reproduction, Fertility and Development 12: 319-327.

McCoard SA, Koolaard J, Charteris A, Luo D (2010) Brief communication: Effect of twinning and sex on carcass weight and composition in lambs. Proceedings of the New Zealand Society of Animal Production 70: $133-136$.

Milton JS (2001) Estadística para biología y ciencias de la salud 3ra. Edición. Interamericana Mc Graw-Hill. México. 722p.

Molina A, Gallego L, Sotillo JL (1991) Evolución anual del peso vivo y de la nota de condición corporal de ovejas de la raza Manchega en diferentes estados productivos. Archivos de Zootecnia 40: 237-249.

NRC (2007) Nutrient Requirements of Small Ruminants Sheep, Goats, Cervids, and New World Camelids. The National Academies Press. Washington, DC. USA. pp: 244-270.

Owens FN, Dubeski P, Hanson CF (1993) Factors that alter the growth and development of ruminants. Journal of Animal Science 71: 3138-3150.

Partida JA, Braña D, Martínez L (2009) Desempeño productivo y propiedades de la canal de ovinos Pelibuey y sus cruzas con Suffolk o Dorset. Técnica Pecuaria en México 47: 313-322.

Partida JA, Martínez L (2010) Composición corporal de corderos Pelibuey en función de la concentración energética de la dieta y del peso al sacrificio. Veterinaria México 41: 177-190.

Partida JA, Braña D (2011) Metodología para la evaluación de la canal ovina. Centro Nacional de Investigación Disciplinaria en Fisiología Animal. Instituto Nacional de Investigaciones Forestales, Agrícolas y Pecuarias, México. 58p.

Peña F, Cano T, Domenech V, Alcalde MJ, Martos J, García-Martínez A, et al. (2005) Influence of sex, slaughter weight and carcass weight on "non-carcass" and carcass quality in Segureña lambs. Small Ruminant Research 60: 247-254.

Pérez P, Maino M, Tomic G, Mardones E, Pokniak J (2002) Carcass characteristics and meat quality of Suffolk Down suckling lambs. Small Ruminant Research 44: 233-240.

Pérez P, Maino M, Morales MS, Tomic G, Aguilera F, Egaña Jl (2012) Meat quality and carcass characteristics of Merino Precoce suckling lambs raised under confinement in the Mediterranean semi-humid dryland of Central Chile. Ciencia e Investigación Agraria 39: 289-298. 
Ramsey WS, Hatfield PG, Wallace JD (1998) Relationships among ewe milk production and ewe and lamb forage intake in Suffolk and Targhee ewes nursing single or twin lambs. Journal of Animal Science 76: 1247-1253.

Rajab MH, Cartwright TC, Dahm PF, Figueiredo EA (1992) Performance of three tropical hair sheep breeds. Journal of Animal Science 70: 3351-3359.

Ríos-Utrera A, Calderón-Robles R, Lagunes-Lagunes J, Oliva-Hernández J (2014) Ganancia de peso predestete en corderos Pelibuey y sus cruces con Blackbelly, Dorper y Katahdin. Nova Scientia 6: 272-286.

Sales F, Pacheco D, Blair H, Kenyon P, McCoard S (2013) Muscle free amino acid profiles are related to differences in skeletal muscle growth between single and twin ovine fetuses near term. SpringerPlus 2: 483p.

Sanson DW, West TR, Tatman WR, Riley ML, Judkins MB, Moss GE (1993) Relationship of body composition of mature ewes with condition score and body weight. Journal of Animal Science 71: 1112-1116.

SAS (2002) SAS/STAT User's Guide (Release 9.0). Cary, North Carolina, USA: SAS Institute.

Snowder GD, Glimp HA (1991) Influence of breed, number of suckling lambs, and stage of lactation on ewe milk production and lamb growth under range conditions. Journal of Animal Science 69: 923-930.

Teke B, Akdag F (2012) The effects of age of lamb and parity of dam and sex and birth type of lamb on suckling behaviours of Karayaka lambs. Small Ruminant Research 103: 176-181.

Thompson J, Meyer H (1994) Body condition scoring of sheep. Oregon State University Extension Service. $4 p$.

Wu G (2009) Amino acids: metabolism, functions, and nutrition. Amino Acids 37: 1-17. 
